\title{
Microscopic study of neutrino trapping in hyperon stars
}

\author{
I. Vidaña ${ }^{1}$, I. Bombaci ${ }^{1}$, A. Polls ${ }^{2}$, and A. Ramos ${ }^{2}$ \\ 1 Dipartimento di Fisica, Università di Pisa and INFN Sezione di Pisa,Via Buonarroti 2, 56127 Pisa, Italy \\ 2 Departament d'Estructura i Constituents de la Matèria, Universitat de Barcelona, 08028 Barcelona, Spain
}

\author{
Received 4 September 2002 / Accepted 29 November 2002
}

\begin{abstract}
Employing the most recent parametrization of the baryon-baryon interaction of the Nijmegen group, we investigate, in the framework of the Brueckner-Bethe-Goldstone many-body theory at zero temperature, the influence of neutrino trapping on the composition, equation of state, and structure of neutron stars, relevant to describe the physical conditions of a neutron star immediately after birth (protoneutron star). We find that the presence of neutrinos changes significantly the composition of matter delaying the appearance of hyperons and making the equation of state stiffer. We explore the consequences of neutrino trapping on the early evolution of a neutron star and on the nature of the final compact remnant left by the supernova explosion.
\end{abstract}

Key words. dense matter - equation of state - stars: neutron

\section{Introduction}

Neutrinos play a crucial role in the physics of supernova explosions (Janka \& Müller 1996) and in the early evolution of their compact stellar remnants (Burrows \& Lattimer 1986; Janka \& Müller 1995). During the collapse of the pre-supernova core, a large number of neutrinos is produced by electron capture process. Immediately following the core bounce the radius of the newly formed neutron star shrinks from about $100 \mathrm{~km}$ to about $10 \mathrm{~km}$. During this same period (up to about $1 \mathrm{~s}$ after core bounce) substantial matter accretion occurs on the compact star (this accretion may eventualy led to the formation of a black hole). As the newly formed neutron star contracts the neutrino mean free path $\lambda_{v}$ decreases, and above a critical value of the density (neutrino trapping density) $\lambda_{v}$ becomes smaller than the stellar radius. Under these physical conditions neutrinos are trapped in the star, i.e., the neutrino diffusion time is of the order of a few tens of seconds. Neutrino trapping has a strong influence on the overal stiffness of the equation of state (EoS) of dense stellar matter. Thus, the physical conditions of the hot and lepton-rich newborn neutron star (the so-called protoneutron star) differ substantially from those of the cold and deleptonized neutron star. Nevertheless, this stage nearly fulfills the conditions of hydrostatical equilibrium (Burrows \& Lattimer 1986).

The composition and the structure of protoneutron stars have been systematically investigated by Prakash et al. (1997) and by Strobel et al. (1999) using a large sample of modern equations of state of dense stellar matter. The implications of the early evolution of a protoneutron star on the concept of neutron star maximum mass have been studied by the authors of
Refs. (Bombaci 1996; Prakash et al. 1997; Strobel \& Weigel 2001).

Due to the rapid increase of the nucleon chemical potentials with density, hyperons $\left(\Lambda, \Sigma^{-}, \Sigma^{0}, \Sigma^{+}, \Xi^{-}\right.$and $\Xi^{0}$ particles) are expected to appear in the core of neutron stars, as suggested in the pioneer work by Ambartsumyan \& Saakyan (1960). Since then the structrural properties of these hyperon stars have been studied by many researchers using a variety of aproaches (see e.g., Pandharipande 1971; Glendenning 1985; Keil \& Janka 1994; Shaffner \& Mishustin 1996; Prakash et al. 1997; Balberg \& Gal 1997; Baldo et al. 2000; Vidaña et al. 2000a)

All the previous studies of hyperonic matter with trapped neutrinos have been done in the framework of a relativistic theoretical field model of nucleons and hyperons interacting via meson exchange in a mean field approximation (Keil \& Janka 1994; Prakash et al. 1997). In the present work, we use a microscopic approach instead, which is based on the BruecknerBethe-Goldstone (BBG) many body theory. In our calculations the basic input is the baryon-baryon interaction for the complete baryon octet $\left(n, p, \Lambda, \Sigma^{-}, \Sigma^{0}, \Sigma^{+}, \Xi^{-} \& \Xi^{0}\right)$ developed recently by Stoks \& Rijken (1999). Within this approach we compute the EoS of hyperonic matter with trapped neutrinos and the corresponding properties of newborn hyperon stars. A similar microscopic approach has been recently employed by Baldo et al. (2000) and Vidaña et al. (2000a) to study cold and deleptonized hyperon stars. The primery purpose of the present work is to investigate the effects of neutrino trapping on the structure and evolution of newly formed hyperon stars.

The paper is organized in the following way. A brief review of the Brueckner-Hartree-Fock (BHF) approximation of 
the BBG many-body theory at zero temperature extended to the hyperonic sector is given in Sect. 2.1. Equilibrium conditions and EoS of $\beta$-stable matter are discussed in Sect. 2.2. Section 3 is devoted to the presentation and discussion of the results. Finally, a short summary and the main conclusions of this work are drawn in Sect. 4.

\section{Equation of State and equilibrium conditions}

\subsection{Many-body theory of hyperonic matter}

Our calculation of the EoS of high density matter is based on the BHF approximation of the BBG many-body theory at zero temperature extended to the hyperonic sector (Baldo et al. 2000; Vidaña et al. 2000a). We start it by constructing all baryon-baryon (nucleon-nucleon (NN), hyperon-nucleon (YN) and hyperon-hyperon (YY)) $G$-matrices, which describe in an effective way the interactions between baryons in the presence of a surrounding hadronic medium. They are formally obtained by solving the well known Bethe-Goldstone equation, written schematically as

$$
\begin{aligned}
G(\omega)_{B_{1} B_{2}, B_{3} B_{4}}= & V_{B_{1} B_{2}, B_{3} B_{4}} \\
& +\sum_{B_{5} B_{6}} V_{B_{1} B_{2}, B_{5} B_{6}} \frac{Q_{B_{5} B_{6}}}{\omega-E_{B_{5}}-E_{B_{6}}+i \eta} \\
& \times G(\omega)_{B_{5} B_{6}, B_{3} B_{4}}
\end{aligned}
$$

In the expression above the first (last) two subindices indicate the initial (final) two-baryon states compatible with a given value $S$ of the strangeness (NN for $S=0$, YN for $S=-1,-2$, and YY for $S=-2,-3,-4), V$ is the bare baryon-baryon interaction, $Q$ is the Pauli operator which allows only intermediate two-body states compatible with the Pauli principle, and $\omega$ is the so-called starting energy.

The single-particle energy of a baryon $B_{i}$ is given by (we use units in which $\hbar=1, c=1$ )

$E_{B_{i}}=M_{B_{i}}+\frac{k^{2}}{2 M_{B_{i}}}+U_{B_{i}}(k)$,

where $M_{B_{i}}$ denotes the rest mass of the baryon, and the singleparticle potential energy $U_{B_{i}}(k)$ represents the averaged field "felt" by the baryon due to its interaction with the other baryons of the medium. In the BHF approximation $U_{B_{i}}(k)$ is given by

$U_{B_{i}}(k)=\operatorname{Re} \sum_{B_{j}} \sum_{k^{\prime} \leq k_{F_{B_{j}}}}\left\langle\boldsymbol{k} \boldsymbol{k}^{\prime}\left|G_{B_{i} B_{j}, B_{i} B_{j}}\left(\omega=E_{B_{i}}+E_{B_{j}}\right)\right| \boldsymbol{k} \boldsymbol{k}^{\prime}\right\rangle$,

where a sum over all the Fermi seas of the different baryon species is performed, and the matrix elements are properly antisymmetrized when baryons $B_{i}$ and $B_{j}$ belong to the same isomultiplet. We note here that Brueckner-type calculations are very time consuming since one has to solve a self-consistent set of coupled-channel equations for the different strangeness sectors (see Vidaña et al. 2000b for details). Therefore, in order to do the calculations less time consuming we have adopted the so-called discontinuous prescription for the single-particle energy (i.e., $E_{B_{i}}=M_{B_{i}}+k^{2} / 2 M_{B_{i}}$ for $k>k_{F_{B_{i}}}$ ) when solving the Bethe-Goldstone equation. The present calculations have been carried out by using the most recent parametrization of the bare baryon-baryon potential for the complete baryon octet as defined by Stoks \& Rijken (1999). This potential model, which aims at describing all interaction channels with strangeness from $S=0$ to $S=-4$, is based on $S U(3)$ extensions of the Nijmegen nucleon-nucleon and hyperon-nucleon potentials (Rijken et al. 1998).

Once a self-consistent solution of Eqs. (1)-(3) is achieved, the baryonic energy density $\varepsilon_{\mathrm{b}}$ can be evaluated in the BHF approximation according to the following expression:

$$
\varepsilon_{\mathrm{b}}=2 \sum_{B_{i}} \int_{0}^{k_{F_{B_{i}}}} \frac{\mathrm{d}^{3} k}{(2 \pi)^{3}}\left(M_{B_{i}}+\frac{k^{2}}{2 M_{B_{i}}}+\frac{1}{2} U_{B_{i}}^{N}(k)+\frac{1}{2} U_{B_{i}}^{Y}(k)\right),
$$

where we have split, according to Eq. (3) the baryon singleparticle potential $U_{B_{i}}$ into a contribution, $U_{B_{i}}^{N}$, coming from the interaction of the baryon $B_{i}$ with all the nucleons of the system, and a contribution, $U_{B_{i}}^{Y}$, coming from the interaction with the hyperons.

It is well known that non-relativistic many-body calculations, based on purely two-body forces, fail to reproduce the empirical saturation point for symmetric nuclear matter and the binding energy and radius of light nuclei. The remedy to the previous deficiency is to introduce three-body forces (TBF) between nucleons. In hyperonic matter the repulsion induced at high densities by nucleon three-body forces enhances substancially the hyperon population which in turn induces a strong softening of the EoS (Schulze et al. 1998; Baldo et al. 2000).

In order to include the effects of TBF between nucleons in our computational scheme, we have replaced the pure nucleonic contribution to the baryonic energy density $\varepsilon_{\mathrm{b}}$ (Eq. (4)), i.e.,

$\varepsilon_{N N} \equiv 2 \sum_{N_{i}} \int_{0}^{k_{F_{N_{i}}}} \frac{\mathrm{d}^{3} k}{(2 \pi)^{3}}\left(M_{N_{i}}+\frac{k^{2}}{2 M_{N_{i}}}+\frac{1}{2} U_{N_{i}}^{N}(k)\right)$,

by the analytic parametrization developed by Heiselberg \& Hjorth-Jensen (1999)

$\varepsilon_{N N}=\rho_{N}\left(M_{N}+E_{0} u \frac{u-2-\delta}{1+u \delta}+S_{0} u^{\gamma}\left(1-2 Y_{p}\right)^{2}\right)$.

Here $u=\rho_{N} / \rho_{0}$ is the ratio of the nucleonic density to nuclear saturation density $\left(\rho_{0}=0.16 \mathrm{fm}^{-3}\right)$ and $Y_{p}=\rho_{p} / \rho_{N}$ is the proton fraction. This approach parametrizes the nucleon energy density obtained from the variational calculation using the Argonne $V_{18}$ nucleon-nucleon interaction with threebody forces and relativistic boost corrections of Akmal et al. (1998). The best fit of this simple functional is obtained for $E_{0}=15.8 \mathrm{MeV}, S_{0}=32 \mathrm{MeV}, \gamma=0.6$ and $\delta=0.2$ (see Heiselberg \& Hjorth-Jensen 1999 for more details). Therefore the baryonic energy density will be given by

$\varepsilon_{\mathrm{b}}=\varepsilon_{N N}+\varepsilon^{\prime}$,

with $\varepsilon_{N N}$ obtained from Eq. (6) and

$$
\begin{aligned}
\varepsilon^{\prime}= & 2 \sum_{N_{i}} \int_{0}^{k_{F_{N_{i}}}} \frac{\mathrm{d}^{3} k}{(2 \pi)^{3}} \frac{1}{2} U_{N_{i}}^{Y}(k)+2 \sum_{Y_{i}} \int_{0}^{k_{F_{Y_{i}}}} \frac{\mathrm{d}^{3} k}{(2 \pi)^{3}} \\
& \times\left(M_{Y_{i}}+\frac{k^{2}}{2 M_{Y_{i}}}++\frac{1}{2} U_{Y_{i}}^{N}(k)+\frac{1}{2} U_{Y_{i}}^{Y}(k)\right) .
\end{aligned}
$$




\subsection{Equilibrium conditions and EoS of $\beta$-stable matter}

The concentrations of the different constituents in the stellar interior are determined by the requirements of electric charge neutrality and equilibrium under weak interaction processes ("chemical" equilibrium)

$B_{1} \rightarrow B_{2}+\ell+\bar{v}_{\ell}, \quad B_{2}+\ell \rightarrow B_{1}+v_{\ell}$

where $B_{1}$ and $B_{2}$ are baryons, and $\ell$ is a lepton $\left(e^{-}\right.$or $\left.\mu^{-}\right)$and $v_{\ell}$ $\left(\bar{v}_{\ell}\right)$ is the associated neutrino (antineutrino). For stellar matter with trapped neutrinos, these two requirements imply that the relations

$\sum_{i} \rho_{B_{i}}^{(+)}+\sum_{\ell} \rho_{\ell}^{(+)}=\sum_{i} \rho_{B_{i}}^{(-)}+\sum_{\ell} \rho_{\ell}^{(-)}$

$\mu_{i}=b_{i} \mu_{\mathrm{n}}-q_{i}\left(\mu_{\ell}-\mu_{v_{\ell}}\right)$,

are satisfied. Above, $\rho_{B_{i}}\left(\rho_{\ell}\right)$ denotes the baryon (lepton) number density and the superscripts $( \pm)$ on $\rho_{B_{i}}\left(\rho_{\ell}\right)$ signify positive or negative electric charge. The symbol $\mu_{i}$ refers to the chemical potential of baryon of the species $i, b_{i}$ is its baryon number, and $q_{i}$ is its charge. The chemical potential of the neutron is denoted by $\mu_{\mathrm{n}}$, and the chemical potential of the neutrino $v_{\ell}$ is denoted by $\mu_{v_{\ell}}$. Because neutrinos are trapped in the star, the lepton number per baryon $Y_{L \ell}$ of each lepton flavor must be conserved on dynamical time scales

$Y_{\mathrm{Le}}=Y_{\mathrm{e}}+Y_{v_{\mathrm{e}}}, \quad Y_{L \mu}=Y_{\mu}+Y_{v_{\mu}}$

Gravitational collapse calculations of the core of massive stars indicate that at the onset of trapping the electron lepton fraction $Y_{\mathrm{Le}}=Y_{\mathrm{e}}+Y_{v_{\mathrm{e}}} \approx 0.4$. In addition, as the trapping in supernova occurs when the collapsing core reaches densities where no muons exist, we can impose $Y_{L \mu}=Y_{\mu}+Y_{v_{\mu}}=0$.

For matter where nucleons and hyperons are the relevant hadronic degrees of freedom the chemical equilibrium conditions can be explicitly written as

$\mu_{\Xi^{-}}=\mu_{\Sigma^{-}}=\mu_{\mathrm{n}}+\mu_{\mathrm{e}}-\mu_{v_{\mathrm{e}}}$,

$\mu_{\Lambda}=\mu_{\Xi^{0}}=\mu_{\Sigma^{0}}=\mu_{\mathrm{n}}$,

$\mu_{\Sigma^{+}}=\mu_{\mathrm{p}}=\mu_{\mathrm{n}}-\mu_{\mathrm{e}}+\mu_{v_{\mathrm{e}}}$,

$\mu_{\mu}-\mu_{v_{\mu}}=\mu_{\mathrm{e}}-\mu_{v_{\mathrm{e}}}$.

In the case of neutrino-free matter (relevant to describe the cold and deleptonized neutron star) the new equilibrium conditions can be obtained from the previous equations simply by taking $\mu_{v_{\mathrm{e}}}=\mu_{v_{\mu}}=0$.

For a given value of the total baryon number density

$\rho_{b}=\sum_{i} \rho_{B_{i}}$

the composition of stellar matter, i.e., the baryonic $\left(Y_{B_{i}}=\right.$ $\left.\rho_{B_{i}} / \rho_{\mathrm{b}}\right)$ and leptonic $\left(Y_{\ell_{i}}=\rho_{\ell_{i}} / \rho_{\mathrm{b}}\right)$ fractions of each constituent species, is obtained by solving Eqs. (10), (12) and (13). We will refer to this status of the stellar matter as $\beta$-stable matter.

The chemical potentials of the different particles are the fundamental ingredients when solving the equilibrium conditions summarized in Eq. (13). In the BHF approximation the chemical potentials of the baryons are taken to be equal to the value of the single-particle energy at the Fermi momentum,

$\mu_{B_{i}}=E_{B_{i}}\left(k_{F_{B_{i}}}\right)=M_{B_{i}}+\frac{k_{F_{B_{i}}}^{2}}{2 M_{B_{i}}}+U_{B_{i}}^{N}\left(k_{F_{B_{i}}}\right)+U_{B_{i}}^{Y}\left(k_{F_{B_{i}}}\right)$.

In order to be consistent with our calculation of the baryonic energy density (see the discussion above in connection to the role of nucleon TBF for the saturation properties of nuclear matter), in the case of nucleons, we replace in the chemical potentials given by Eq. (15) the nucleonic BHF contribution $\mu_{N}^{N} \equiv M_{N}+k_{F_{N}}^{2} / 2 M_{N}+U_{N}^{N}\left(k_{F_{N}}\right)$ by $\mu_{N}^{N}=\partial \varepsilon_{N N} / \partial \rho_{N}$. Here $\varepsilon_{N N}$ denotes the parametrization of the nucleonic energy density contribution due to Heiselberg \& Hjorth-Jensen (1999) (see Eq. (6)). For the hyperons, however, we keep the prescription of Eq. (15). The chemical potentials of leptons are calculated using the expressions for non-interacting relativistic fermions which are well known from textbooks.

Once the composition of $\beta$-stable matter is determined we can compute the total energy density $\varepsilon=\varepsilon_{\mathrm{b}}+\varepsilon_{\ell}$, the baryonic pressure using the thermodynamic relation

$P_{\mathrm{b}}=\rho_{\mathrm{b}} \frac{\partial \varepsilon_{\mathrm{b}}}{\partial \rho_{\mathrm{b}}}-\varepsilon_{\mathrm{b}}$

and finally the total pressure $P=P_{\mathrm{b}}+P_{\ell}$. Once again the leptonic contributions to the energy density and pressure are those of a relativistic free Fermi gas.

We note here that, although the hyperon chemical potentials are evaluated according to Eq. (15), and the hyperonic contribution to the nucleon chemical potentials is keep to be $U_{N}^{Y}\left(k_{F_{N}}\right)$, the thermodynamic relation

$\varepsilon+P=\sum_{i=B, L} \rho_{i} \mu_{i}$

is fulfilled within $1 \%$ at saturation density, and within $10 \%$ at the central density corresponding to the maximum mass configuration.

\section{Results}

The composition of $\beta$-stable stellar matter calculated as described in the previous section, is shown in Fig. 1 as a function of the total baryon number density. The upper panel of the figure exhibits the results for neutrino-trapped matter (with $Y_{\mathrm{Le}}=0.4$ and $Y_{\mathrm{L} \mu}=0$ ), whereas the lower panel shows the composition of neutrino-free matter. To begin with, let us comment our results for the composition of neutrino-free matter in connection to the role of the hyperon-nucleon and hyperonhyperon interactions (see Vidaña et al. 2000a, for a more detailed discussion). Firstly, note that although the $\Lambda$ hyperon is about $80 \mathrm{MeV}$ less massive than the $\Sigma^{-}$one, the latter appears at a lower baryon number density. The reason is that the process $e^{-}+n \rightarrow \Sigma^{-}+v_{\mathrm{e}}$ removes both an energetic neutron and an energetic electron, whereas the weak strangeness nonconserving decay of a neutron into a $\Lambda$, being neutral, removes only an energetic neutron. Since the electron chemical potential in matter is larger than the mass difference $M_{\Sigma^{-}}-M_{\Lambda}$, the condition for the onset of the $\Sigma^{-}, \mu_{\mathrm{n}}+\mu_{\mathrm{e}}=\mu_{\Sigma^{-}}$, is fulfilled 


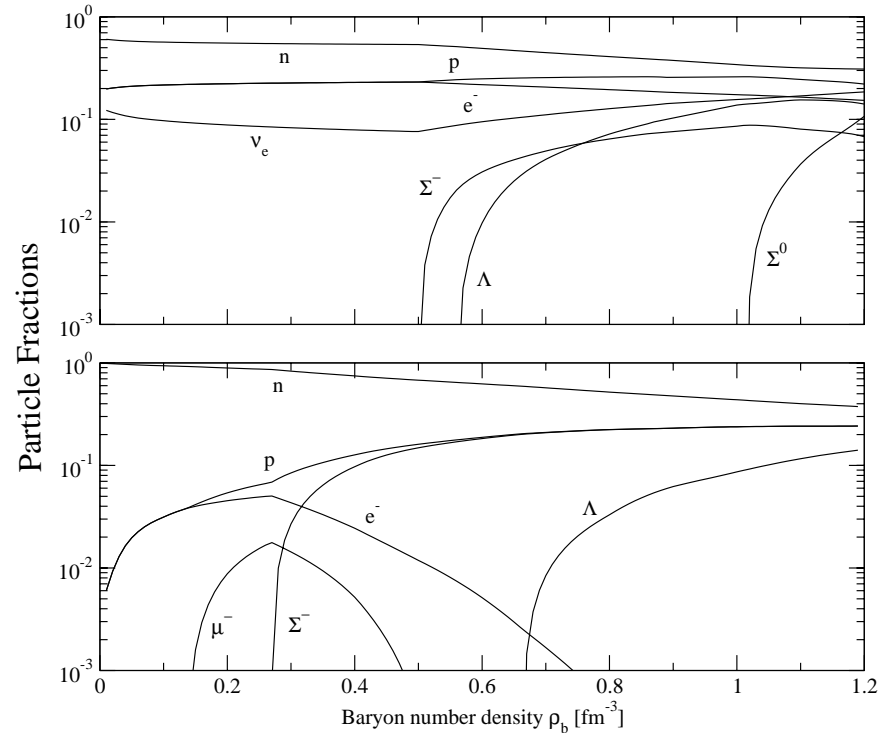

Fig. 1. Compositon of $\beta$-stable hyperonic matter as a function of the baryon number density. Upper panel show results for the neutrinotrapped case (with $Y_{\mathrm{L}_{\mathrm{e}}}=0.4$ and $Y_{\mathrm{L}_{\mu}}=0$ ), whereas those for neutrinofree matter are reported in the lower one.

at lower densities than the corresponding one for the appeareance of the $\Lambda, \mu_{\mathrm{n}}=\mu_{\Lambda}$. Furthermore, as soon as the $\Sigma^{-}$appears it becomes energetically more favorable for the system to keep charge neutrality with $\Sigma^{-}$hyperons than with leptons, therefore the lepton concentrations begin to fall. The onset of $\Lambda$ formation takes place at higher baryon number density as soon as the chemical potential of the neutron equals that of the $\Lambda$. No other hyperons appear at baryon number densities below $\rho_{\mathrm{b}}=1.2 \mathrm{fm}^{-3}$ within our many-body approach.

Having in mind these results as a reference, let us now consider the effect of neutrino trapping. As it can be seen from the upper panel of Fig. 1 the composition of matter is significantly altered when neutrinos are trapped. The first thing to notice is that trapping keeps the electron concentration high so that matter is more proton rich in comparison with the case in which neutrinos have diffused out. Notice in addition that muons are not present, and the onset of hyperon formation is changed. The appearance of the $\Sigma^{-}$hyperon is now governed by $\mu_{\Sigma^{-}}=\mu_{\mathrm{n}}+\mu_{\mathrm{e}}-\mu_{v_{\mathrm{e}}}$, whereas in the neutrino-free case the condition to be fulfilled was $\mu_{\Sigma^{-}}=\mu_{\mathrm{n}}+\mu_{\mathrm{e}}$. Due to the fact that $\mu_{\mathrm{e}}-\mu_{v_{\mathrm{e}}}$ is much smaller than $\mu_{\mathrm{e}}$, the appearance of the $\Sigma^{-}$occurs at a higher baryon number density $\left(\rho_{\mathrm{b}} \approx 0.50 \mathrm{fm}^{-3}\right)$, and the amount of $\Sigma^{-}$'s is smaller. This, in turn, implies less $\Sigma^{-} n$ pairs. Since the $\Sigma^{-} n$ interaction is attractive in this model (see, e.g., Fig. 7 of Vidaña et al. 2000b) the chemical potential of the neutrons becomes less attractive. As a consequence, the $\Lambda$ and $\Sigma^{0}$ (which in neutrino-free matter was not present) hyperons appear at a lower densities $\left(\rho_{\mathrm{b}} \approx 0.57 \mathrm{fm}^{-3}\right.$ and $\rho_{\mathrm{b}} \approx 1.02 \mathrm{fm}^{-3}$, respectively). Finally, the neutrino fraction, which initially decreases with baryon number density in order to keep $Y_{\mathrm{Le}}$ constant, begins to increase as soon as $\Sigma^{-}$'s are present on the system due to the formation of this baryon through the process $e^{-}+n \rightarrow \Sigma^{-}+v_{\mathrm{e}}$.
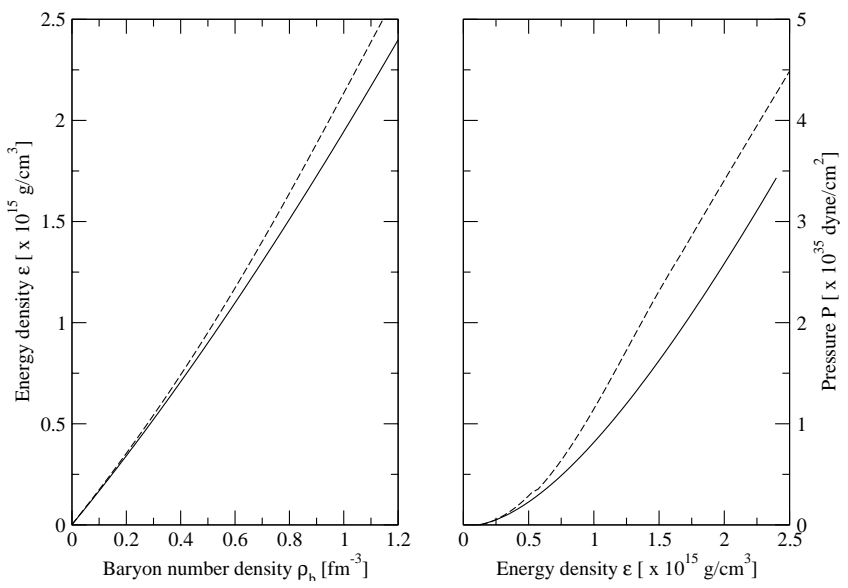

Fig. 2. Total energy density $\varepsilon$ as a function of the baryon number density $\rho_{\mathrm{b}}$ (left panel) and total pressure as a function of $\varepsilon$ (right panel) for $\beta$-stable neutron star matter. Solid lines in both panels show results for neutrino-free matter, whereas results for the neutrino-trapped case correspond to dashed lines.

Let us now examine the effect of neutrino trapping on the EoS for $\beta$-stable neutron star matter. We show in Fig. 2 the results for the total energy density $\varepsilon$ versus the baryon number density (left panel) and the total pressure as a function of $\varepsilon$ (right panel). The dashed lines represent the results for neutrino-trapped matter whereas the solid lines show the result for neutrino-free matter. As we can see the EoS for neutrinotrapped matter is stiffer than that for neutrino-free matter. This result is a consequence of the different composition of stellar matter in the two cases illustrated in Fig. 1. In addition, it is interesting to note that, even in those regions where nucleons are the only relevant baryonic degrees of freedom (i.e., up to $\rho_{\mathrm{b}} \sim 0.50 \mathrm{fm}^{-3}$ ), the EoS for neutrino-trapped matter is stiffer than the one for neutrino-free matter. In fact, the extra leptonic pressure caused by neutrino-trapping is greater than the decrease in pressure of nucleons induced by the reduction of the nuclear symmetry energy in the proton rich matter with trapped neutrinos (compare the the proton abundances in the upper and lower panel of Fig. 1 for $\rho_{\mathrm{b}}<0.50 \mathrm{fm}^{-3}$ ) (Chiapparini et al. 1996; Prakash et al. 1997). This can be seen in Fig. 3 where we plot the baryonic (left panel) and leptonic (right panel) contributions to the total pressure for neutrino-free (solid lines) and neutrino-trapped (dashed lines) matter.

Finally, let us consider the effect of neutrino trapping on the properties of neutron stars. To this end, we have solved the well known Tolman-Oppenheimer-Volkov equations for the structure of non-rotating stellar configurations in general relativity. To describe the stellar crust we used the equations of state by Feynman-Metropolis-Teller (Feynman et al. 1949), Baym-Pethick-Sutherland (Baym et al. 1971) and Negele-Vautherin (1973). In Fig. 4 we show the resulting stellar equilibrium sequences. In the left panel we plot the gravitational mass $M_{\mathrm{G}}$ in units of the solar mass $\left(M_{\odot}=1.989 \times 10^{33} \mathrm{~g}\right)$ as a function of the central energy density, while in the right panel $M_{\mathrm{G}}$ is plotted as a function of the stellar radius $R$. Dashed (solid) lines represent the results for neutrino-trapped (neutrino-free) matter. The properties of 

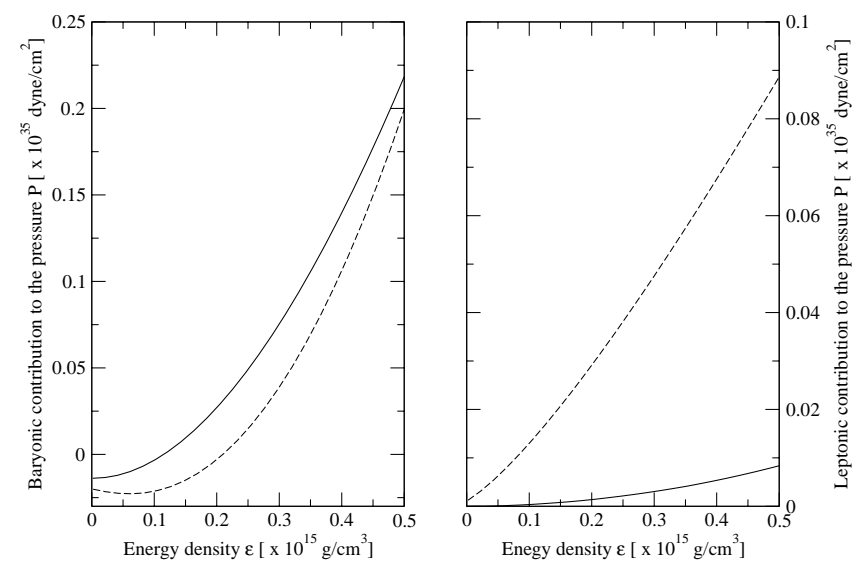

Fig. 3. Baryonic (left panel) and leptonic (right panel) contributions to the total pressure $P$ as a function of the total energy density density $\varepsilon$ for the two scenarios considered: neutrino-free (solid lines) and neutrino-trapped (dashed lines) matter. The baryon number density corresponding to the maximum energy density plotted is $\rho_{\mathrm{b}} \sim$ $0.3 \mathrm{fm}^{-1}$, at which the only relevant hadronic degrees of freedom are nucleons.

the maximum mass configurations are summarized in Table 1. The EoS for neutrino-free matter calculated within the present microscopic approach (Baldo et al. 2000; Vidaña et al. 2000a) gives a maximum mass below $\sim 1.44 M_{\odot}$, in conflict with measured neutron star masses. This means that our EoS with hyperons needs to be stiffer. Within a microscopic approach, as the one used in this work, one should try to trace the origin of this problem back to the underlying hyperon-nucleon and hyperon-hyperon two-body interaction, or to the possible repulsive three-body forces involving one or more hyperons (i.e., YNN, YYN or YYY), not included in this work and similar studies. Unfortunally, the YY two-body interaction is not well constrained at present due to the scarce amount of experimental data, and although active research is devoted to the construction of three-body forces between nucleons and hyperons they are not yet available. On the other hand the neglect of the hyperonic degrees of freedom on the dense matter EoS always leads to an unrealistic overstimate of the stellar maximum mass.

In agreement with previous studies we find that the maximum mass supported by neutrino-trapped EoS is larger than the corresponding one supported by neutrino-free matter EoS. The overall effect of neutrino trapping on the maximum mass configuration is opposite in the case of matter in which the only baryonic degrees of freedom considered are nucleons (Bombaci 1996; Prakash et al. 1997). In the latter case, the lost of leptonic pressure when neutrinos are diffused out of the star is smaller than the gain in baryonic pressure arising from the nuclear symmetry energy due to the decrease in the number of protons. As a consequence, in nucleonic $\beta$-stable matter, the maximum mass supported by neutrino-free matter is larger than the corresponding one supported by neutrino-trapped matter, as it is shown by our present results reported in the right panel of Fig. 5.

A very important implication of neutrino trapping in dense matter with hyperons is the possibility of having metastable
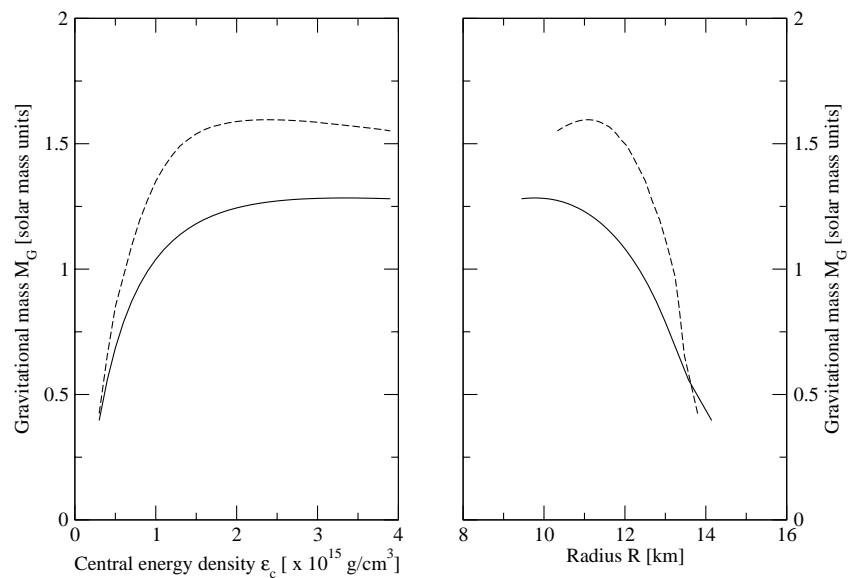

Fig. 4. Gravitational mass as a function of the central energy density (left panel) and radius (right panel) of the star for the two scenarios considered: neutrino-free (solid lines) and neutrino-trapped matter (dashed lines).

neutron stars and a delayed formation of a "low-mass" $\left(M=1-2 M_{\odot}\right)$ black hole. This is illustrated in Fig. 5 where we show the gravitational mass of the star as a function of its baryonic mass $M_{\mathrm{B}}$, which is taken as the total number of baryons in the star times the average nucleon mass. If hyperons are present (left panel), then deleptonization lowers the range of gravitational masses that can be supported by the EoS from about $1.59 M_{\odot}$ to about $1.28 M_{\odot}$ (see dotted horizontal lines in the figure). Since most of the matter accretion on the forming neutron star happens in the very early stages after birth $(t<1 \mathrm{~s})$, with a good approximation, the neutron star baryonic mass stays constant during the evolution from the initial protoneutron star configuration to the final neutrino-free configuration. Then, within our EoS model, protoneutron stars which at birth have a gravitational mass between 1.28-1.59 $M_{\odot}$ (a baryonic mass between $1.40-1.72 M_{\odot}$ ) will be stabilized by neutrino trapping effects long enough to carry out nucleosynthesis accompayning a type-II supernova explosion. After neutrinos leave the star, the EoS is softened and it can not support anymore the star against its own gravity. Thus the newborn neutron star collapses to a black hole (Keil \& Janka 1994; Bombaci 1996; Prakash et al. 1997). A similar qualitative behaviour is expected also in the case in which dense matter contains a Bose-Einstein condensate of negative kaons (Brown \& Bethe 1994; Prakash et al. 1997). On the other hand, if only nucleons are considered to be the relevant baryonic degrees of freedom (right panel), no metastability occurs and a black hole is unlikely to be formed during the deleptonization since the gravitational mass increases during this stage which happens at constant baryonic mass. If a black hole were to form from a star with only nucleons, it is much more likely to form during the post-bounce accretion stage.

To end this section, we show in Fig. 6 the differences of the internal composition as a function of the radial coordinate of a protoneutron star (upper panel) and the corresponding deleptonized neutron star (lower panel) for a constant value, $M_{B}=1.34 M_{\odot}$, of the stellar baryonic mass. The central energy density of the protoneutron star is not high enough to allow 
Table 1. Neutron star properties of the maximum mass configuration for the two scenarios considered: neutrino-trapped and neutrino-free matter. $\varepsilon_{\mathrm{c}}$ denotes the central energy density, $\rho_{b_{\mathrm{c}}}$ the corresponding central baryon number density, $M_{\mathrm{G}}$ the gravitational mass, $M_{\mathrm{B}}$ the baryonic mass, $R$ the radius of the star, $R_{\mathrm{Y}}$ the radius of the hyperonic core, and $\Delta R_{\text {crust }}$ the thickness of the star crust.

\begin{tabular}{|c|c|c|c|c|c|}
\hline Scenario & \multicolumn{2}{|c|}{$\begin{array}{c}\varepsilon_{\mathrm{c}} \\
{\left[\times 10^{15} \mathrm{~g} / \mathrm{cm}^{3}\right]}\end{array}$} & $\begin{array}{c}\rho_{b_{\mathrm{c}}} \\
{\left[\mathrm{fm}^{-3}\right]} \\
\end{array}$ & $\begin{array}{l}M_{\mathrm{G}} \\
{\left[M_{\odot}\right]} \\
\end{array}$ & \multirow{2}{*}{$\begin{array}{c}M_{\mathrm{B}} \\
{\left[M_{\odot}\right]} \\
1.724\end{array}$} \\
\hline Trapped & 2.30 & & 1.066 & 1.595 & \\
\hline \multirow[t]{4}{*}{ Free } & 3.19 & & 1.537 & 1.283 & 1.406 \\
\hline & $\begin{array}{c}R \\
{[\mathrm{~km}]}\end{array}$ & $\begin{array}{c}R_{\mathrm{Y}} \\
{[\mathrm{km}]}\end{array}$ & $\begin{array}{c}\Delta R_{\text {crust }} \\
{[\mathrm{km}]} \\
\end{array}$ & & \\
\hline & 11.14 & 6.32 & 0.66 & & \\
\hline & 9.86 & 7.60 & 0.70 & & \\
\hline
\end{tabular}

for the presence of hyperons and only nucleons, electrons and neutrinos are present in the stellar core. This star has a gravitational mass $M_{\mathrm{G}}=1.28 M_{\odot}$. Nevertheless, as soon as neutrinos diffuse out of the star, pressure decreases, gravity compresses matter, energy density increases and hyperons appear in the star interior. The gravitational mass of the final neutrinofree star is $M_{\mathrm{G}}=1.23 M_{\odot}$. The difference between the initial and final gravitational masses corresponds to the energy which is carried out by neutrinos when they escape from the star. In the present case (i.e., assuming $M_{\mathrm{B}}=1.34 M_{\odot}$ ) this energy is about $9 \times 10^{52} \mathrm{erg}$. In addition, due to the increase of the central energy density, the stellar radius decreases.

\section{Summary and conclusions}

In this paper we have investigated within the framework of the Brueckner-Hartree-Fock approximation the effects of neutrino trapping on the properties of $\beta$-stable neutron star matter including nucleonic and hyperonic degrees of freedom.

We have found that the presence of neutrinos changes significantly the compositon of matter with respect to the neutrino-free case: matter becomes more proton rich, muons are not present, and the appearance of hyperons is moved to higher densities. In additon, the number of strange particles is on average smaller and the EoS stiffer in comparison with the neutrino-free case.

We have found that the value of the maximun mass of hyperon stars decreases as soon as neutrinos diffuse out of the star, contrary to what happens when the only baryonic degrees of freedom considered are nucleons.

Using the microscopic EoS developed in the present work we have found that stars having at birth a gravitational mass between 1.28-1.59 $M_{\odot}$ are metastable, in other words these stellar configurations remain only stable for several seconds (the neutrino trapping time), collapsing afterwards into low-mass black holes.
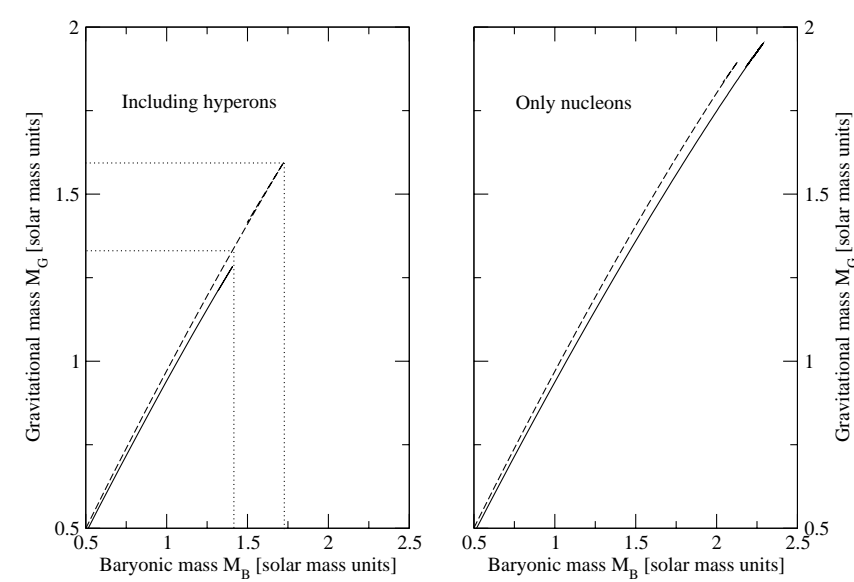

Fig. 5. Gravitational mass as a function of the baryonic mass for the two scenarios considered: neutrino-free (solid lines) and neutrinotrapped matter (dashed lines). Left panel shows results for matter containing nucleons and hyperons as baryonic degrees of freedom, whereas results containing only nucleons are reported on the right one. Dotted lines on the left panel show the window of metastability in the gravitational and baryonic masses.

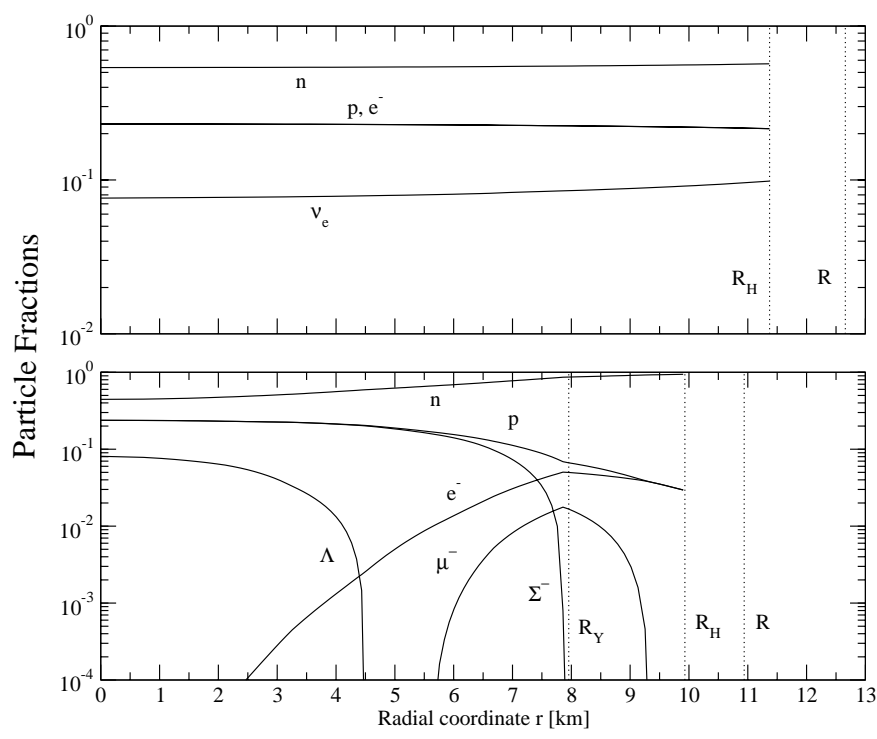

Fig. 6. Internal composition as a function of the radial coordinate of a hyperon star of constant baryonic mass $\left(M_{\mathrm{B}}=1.34 M_{\odot}\right)$ as it evolves from the initial neutrino-trapped (upper panel) to the final neutrinofree (lower panel) configuration. Symbol $R$ indicates the radius of the star, $R_{\mathrm{H}}$ the end of the hadronic core and the beginning of the crust and $R_{\mathrm{Y}}$ the end of the hyperonic core.

\section{References}

Akmal, A., Pandharipande, V. R., \& Ravenhall, D. G. 1998, Phys. Rev. C, 58, 1804

Ambartsumyan, V. A., \& Saakyan G. S. 1960, Soviet Astr., 4, 187

Balberg, S., \& Gal, A. 1997, Nucl. Phys. A, 625, 435

Baldo, M., Burgio, G. F., \& Schulze, H.-J. 2000, Phys. Rev. C, 61, 055801

Baym, G., Pethick, C., \& Sutherland, D. 1971, ApJ, 170, 299

Bombaci, I. 1996, A\&A, 305, 871

Brown, G. E., \& Bethe, H. A. 1994, ApJ, 423, 649 
Burrows, A., \& Lattimer, J. M. 1986, ApJ, 307, 178

Chiapparini, M., Rodrigues, H., \& Duarte, S. B. 1996, Phys. Rev. C, 54,936

Feynman, R., Metropolis, F., \& Teller, E. 1949, Phys. Rev., 75, 1561

Glendenning, N. K. 1985, ApJ, 293, 470

Heiselberg, H., \& Hjorth-Jensen, M. 1999, ApJ, 525, L41; 2000, Phys. Rep., 328, 237

Janka, H.-Th., \& Müller, E. 1995, ApJ, 448, L109

Janka, H.-Th., \& Müller, E. 1996, A\&A, 306, 167

Keil, W., \& Janka, H.-Th. 1994, A\&A, 296, 145

Negele, J. W., \& Vautherin, D. 1973, Nucl. Phys. A, 207, 298

Pandharipande, V. R. 1971, Nucl. Phys. A, 174, 641

Prakash, M., Bombaci, I., Prakash, M., et al. 1997, Phys. Rep., 280, 1
Rijken, Th. A., Stoks, V. G. J., \& Yamammoto, Y. 1998, Phys. Rev. C, 59,21

Schaffner, J., \& Mishustin, I. N. 1996, Phys. Rev. C, 53, 1416

Schulze, H.-J., Baldo, M., Lombardo, U., Cugnon, J., \& Lejeune, A. 1998, Phys. Rev. C, 57, 704; Baldo, M., Burgio, G. F., \& Schulze, H.-J. 1998, ibid., 58, 3688

Stoks, V. G. J., \& Rijken, Th. A. 1999, Phys. Rev. C, 59, 3009

Strobel, K., Schaab, C., \& Weigel, M. K. 1999, A\&A, 350, 497

Strobel, K., \& Weigel, M. K. 2001, A\&A, 367, 582

Vidaña, I., Polls, A., Ramos, A., Engvik, L., \& Hjorth-Jensen, M. 2000a, Phys. Rev. C, 62, 035801

Vidaña, I., Polls, A., Ramos, A., Hjorth-Jensen, M., \& Stoks, V. G. J. 2000b, Phys. Rev. C, 61, 025802 